\title{
The penicillin binding proteins of the genus Haemophilus
}

\author{
P. M. MENDELMAN and D. A. SERFASS*
}

\begin{abstract}
Division of Infectious Diseases, Children's Hospital and Medical Center, 4800 Sand Point Way, NE, PO Box C-5371, and Department of Pediatrics, University of Washington, Seattle, WA 98105, and "Department of Surgery, Temple University, Philadelphia, Pennsylvania 19140, USA
\end{abstract}

\begin{abstract}
Summary. We questioned whether the penicillin binding protein (PBP) profiles of representative strains from the 19 species varied within the genus Haemophilus and whether these profiles would be of taxonomic value. Seventeen of the 19 representative strains studied had distinct PBP profiles; only those of $H$. avium and $H$. paragallinarum were identical. The data support the inclusion of $H$. aegyptius in the genus as a species related to but separate from $H$. influenzae and could not exclude $H$. somnus, $H$. agni, and $H$. equigenitalis from the genus. Comparative PBP analysis within the genus Haemophilus may therefore be useful taxonomically.
\end{abstract}

\section{Introduction}

The genus Haemophilus belongs to the family Pasteurellaceae and its 19 members are aerobic or facultatively anaerobic, nonmotile, pleomorphic, gram-negative rods which require complex media for growth (Kilian and Biberstein, 1984). All are obligate parasites of man (10 species) or other animals ( 9 species) (fig. 1 ) and may be part of the normal flora (Kilian and Biberstein, 1984; Mendelman, 1987). However, all species isolated from man and 6 of 9 species isolated from animals have been associated with clinical disease (Kilian and Biberstein, 1984; Mendelman, 1987; Albritton, 1982); the pathogenic significance of $H$. haemoglobinophilus, $H$. avium, and $H$. paracuniculus has not been determined (Kilian and Biberstein, 1984).

Penicillin binding proteins (PBPs) are chromosomally determined enzymes that participate in cellwall synthesis and covalently bind penicillin. Every bacterium examined contains at least one, and usually several PBPs and they appear to be species specific (Georgopapadakou and Liu, 1980). The PBPs of other genera have been studied in tests with single representative strains from each species (Ohya et al., 1979). In the present study, we investigated whether the PBP profiles of 19 representative strains varied within the genus Haemophilus and if these profiles would be of taxonomic value.

Received 9 Feb. 1988; accepted 22 Feb. 1988.

\section{Materials and methods}

Seventeen isolates were obtained from the American Type Culture Collection (ATCC), and $\boldsymbol{H}$. somnus strain 8025 and $H$. agni were obtained from Dr T. Inzana, Washington State University, Pullman, WA. The following ATCC strains were evaluated: $H$. influenzae 33391, $H$. parainfluenzae $33392, H$. aegyptius $11116, H$. paraphrophilus $29241, H$. haemolyticus $33390, H$. parahaemolyticus $10014, H$. segnis $33393, H$. aphrophilus $33389, H$. paraphrohaemolyticus $29237, H$. ducreyi $33940, H$. haemoglobinophilus 19416, $H$. avium 29546, $H$. paragallinarum 29545, $H$. paracuniculus $29986, H$. parasuis $19417, H$. pleuropneumoniae 27088 , and $H$. equigenitalis 35865 .

All Haemophilus spp. grew on enriched chocolate agar plates (Remel Laboratories, Lenexa, KS, USA) at $37^{\circ} \mathrm{C}$ in $\mathrm{CO}_{2} 5 \%$ in air. $H$. ducreyi required incubation for 4 days in a moist atmosphere and grew better at $33^{\circ} \mathrm{C}$. Initial growth of $H$. avium and $H$. paragallinarum from the ATCC stock vials required Brain Heart Infusion (BHI) Broth (Difco) supplemented with yeast extract $2 \%$, nicotinamide adenine dinucleotide (NAD) (Sigma) 0.01\% and heat-inactivated chicken serum $5 \%$ whereas $H$. ducreyi required the addition of $25 \%$ of defibrinated rabbit blood and incubation at $30-33^{\circ} \mathrm{C}$.

Strains were tested for $\beta$-lactamase production with the chromogenic cephalosporin, nitrocefin, as described by O'Callaghan et al. (1972). Penicillin MIC and MBC determinations were performed by the macro broth dilution method (NCCLS, 1985) in 2-ml volumes of Mueller-Hinton Broth (MHB) supplemented with Iso Vitalex (BBL) $1 \%$ and haemin chloride/histidine (Sigma) $10 \mathrm{mg} / \mathrm{L}$. Inocula were prepared in MHB to a 0.5 McFarland standard and diluted to give a final inoculum of $10^{5} \mathrm{cfu} / \mathrm{ml}$; tests were incubated in $\mathrm{CO}_{2} 5 \%$ in air at $37^{\circ} \mathrm{C}$ for $18-24 \mathrm{~h}$. All Haemophilus spp. were tested in 
this manner with the exception of $\boldsymbol{H}$. ducreyi which was tested by an agar dilution method in GC Base medium (Difco) with Iso Vitalex (BBL) $1 \%$ and haemoglobin $1 \%$ at an inoculum of $10^{4} \mathrm{cfu}$ and with incubation in $\mathrm{CC}_{2} 5 \%$ according to NCCLS guidelines (NCCLS, 1985). The MIC was read as the lowest concentration of penicillin with no visible growth; the $\mathrm{MBC}$ was the concentration of penicillin resulting in $99.9 \%$ killing. Control strains included a $\beta$-lactamase producing strain of $H$. influenzae (RSF 007), the penicillin susceptible $H$. influenzae strain MAP, and Escherichia coli strain ATCC 25922.

PBPs were detected with whole cells grown to mid exponential phase $\left(\mathrm{OD}_{600}=0.6\right)$ in BHI broth, harvested, washed twice with $50 \mathrm{mM}$ Tris- $1 \mathrm{mM} \mathrm{Mg} \mathrm{Cl}$ and $1 \mathrm{ml}$ was concentrated ten-fold (resuspended in $95 \mu \mathrm{l}$ of the above buffer). Five $\mu$ l of $\left[{ }^{3} \mathrm{H}\right]$-penicillin $(0.4 \mathrm{mg} / \mathrm{L})$ was added and incubated at $37^{\circ} \mathrm{C}$ for $15 \mathrm{~min}$, when $5 \mu \mathrm{l}$ of unlabelled $1 \mathrm{M}$ penicillin $\mathrm{G}$ was added. The reaction was stopped with $12.7 \mu \mathrm{l}$ of $20 \%$ Sarkosyl and $22.5 \mu \mathrm{l}$ of sample buffer $(0.0625 \% 2 \mathrm{M}$ Tris, pH 6.8 , SDS $2 \%, \beta$ mercaptoethanol $5 \%$, glycerol $10 \%$, bromophenol blue $0.004 \%$ ). After boiling for $5 \mathrm{~min}, 60 \mu \mathrm{l}$ was loaded into each gel lane. $H$. aphrophilus, $H$. paraphrohaemolyticus, and $H$. ducreyi gew poorly in broth culture and, therefore, were taken directly off agar plates after overnight growth and labelled. As a control, $H$. influenzae strain MAP was labelled similarly. Cells were suspended in the above buffer to an OD of 0.6 and $1 \mathrm{ml}$ of each was concentrated ten-fold and labelled as described. $H$. ducreyi was labelled after growth for 4 days. Electrophoresis and processing were performed as previously described (Mendelman and Chaffin, 1985); the autofluorograms were developed after exposure for 14 days.

\section{Results and discussion}

No strains produced detectable $\beta$-lactamase. The MIC and MBC of penicillin at an inoculum of $10^{5} \mathrm{cfu}$ were $\leqslant 0.5 \mathrm{mg} / \mathrm{L}$ for all strains tested except $H$. parainfluenzae ( $\mathrm{MIC}$ and $\mathrm{MBC}=2 \mathrm{mg} / \mathrm{L}$ ) and $H$. paraphrophilus $(\mathrm{MBC}=1 \mathrm{mg} / \mathrm{L})$.

The PBP pattern of $H$. influenzae strain ATCC 33391 grown in broth to exponential phase (figure,

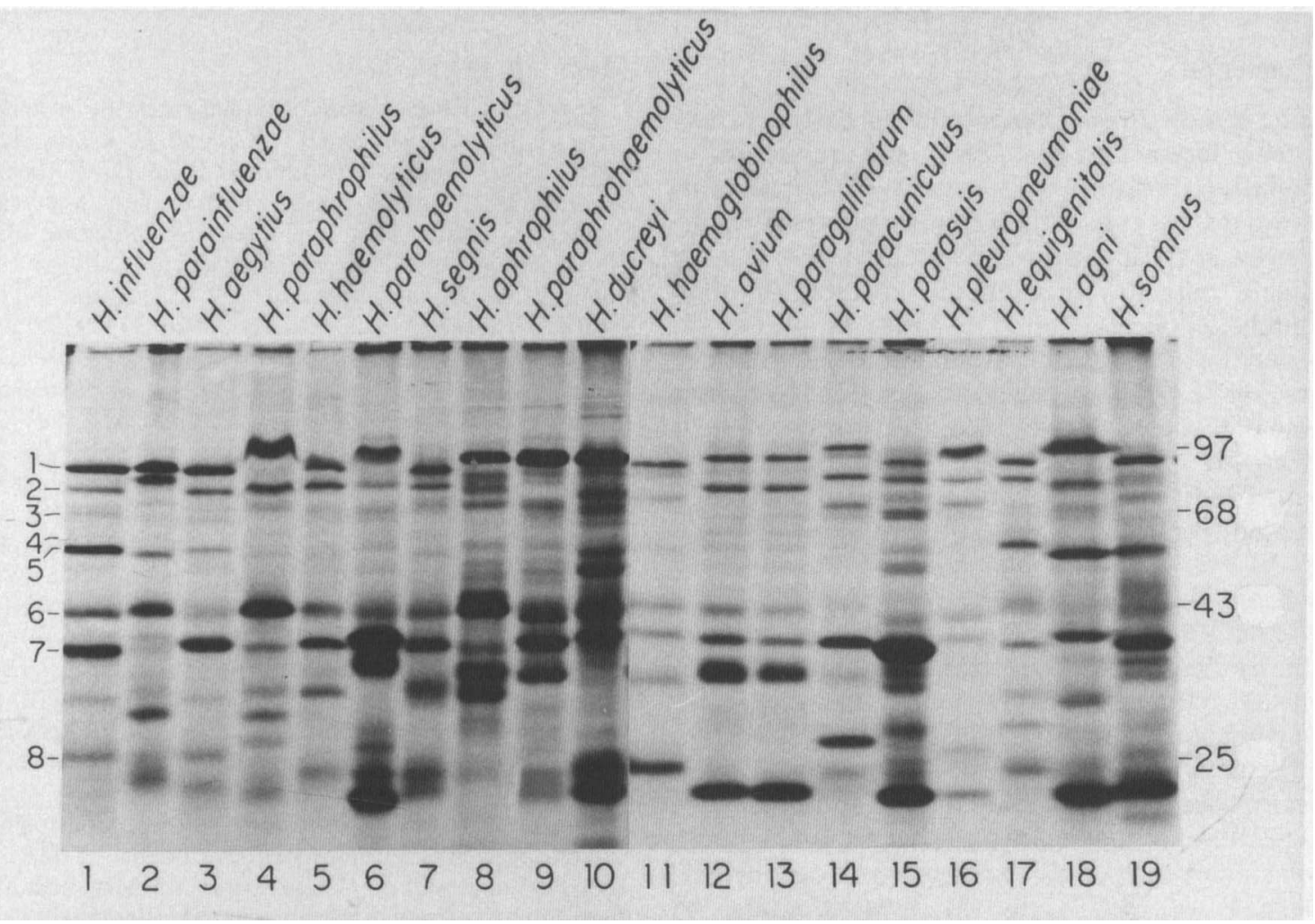

Figure. Autofluorogram of the PBPs of the genus Haemophilus. The 19 species currently listed within the genus Haemophilus (Kilian and Biberstein, 1984), are indicated at the top of each lane. Haemophilus species 1-10 are isolated from man, whereas species 11-19 are isolated (respectively) from dogs, poultry, chickens, rabbits, pigs, pigs, mares, sheep, and cattle (Kilian and Biberstein, 1984; Albritton, 1982; Mendelman, 1987). The numbers to the left of lane 1 indicate the 8 PBPs of the $H$. influenzae strain and the numbers to the right of lane 19 indicate the apparent mol. wt $\left(10^{3}\right)$ of protein standards. Whole cells were incubated with $4 \mathrm{mg} / \mathrm{L}$ (final concentration) of $\left[{ }^{3} \mathrm{H}\right]$-penicillin for $15 \mathrm{~min}$ before SDS-polyacrylamide gel electrophoresis and processing as described in the text. 
lane 1) was identical to the pattern observed with strain MAP taken directly off the agar plate; 8 PBPs were detected (data not shown). Thus, with $H$. influenzae as the control, the PBPs of the 3 strains tested from agar plates appear comparable for analysis with the PBPs of the strains tested from broth cultures. Furthermore, the PBP profile of the $H$. influenzae type strain was identical to that of 10 other susceptible isolates previously described (Mendelman and Chaffin, 1985; Serfass et al. 1986; Mendelman et al., 1987).

Identical PBP profiles were observed for $H$. avium and $H$. paragallinarum (figure, lanes 12 and 13); the other 17 species had distinct PBP profiles. The PBP profile of $H$. influenzae and that of $H$. aegyptius were similar but not identical (figure, lanes 1 and 3 , respectively) and the PBP profiles of $H$. agni and $H$. somnus (figure, lanes 18 and 19 ) and $H$. parainfluenzae, $H$. parahaemolyticus and $H$. paraphrohaemolyticus (figure, lanes 2, 6, and 9) were distinct.

There are certain taxonomic controversies within the genus Haemophilus. It has been proposed that $H$. aegyptius should be represented within biotype III of $H$. influenzae (Carlone et al., 1985; Martel et al., 1985) because no features unequivocally differentiate $H$. aegyptius from $H$. influenzae. DNA homology studies appear to indicate that separation of the two species is unjustified (Martel et al., 1985; Casin et al., 1986); however, $H$. aegyptius has remained as a separate species because it appears to be clinically distinguishable, causing an acute form of contagious conjunctivitis (Kilian and Biberstein, 1984). More recently, it has been associated with Brazilian Purpuric Fever with a high fatality rate (Brazilian Purpuric Fever Study Group, 1987). The PBP patterns of $H$. aegyptius and $H$. influenzae suggest species relatedness, but are distinct; PBP 5 is a major PBP of $H$. influenzae in the 10 penicillin-susceptible isolates reported in the literature including two biotype III strains (Mendelman et al., 1987). In contrast, the comparable PBP (by apparent $\mathrm{M}_{\mathrm{r}}$ ) in the $H$. aegyptius strain studied bound significantly less penicillin (figure, lane 3).

Three species, $H$. somnus, $H$. agni, and $H$. equigenitalis, although described as members of the

\section{REFERENCES}

Albritton W L 1982 Infections due to Haemophilus species other than $H$. influenzae. Annual Review of Microbiology 36: 199216.

Brazilian Purpuric Fever Study Group 1987 Haemophilus genus Haemophilus, are reported not to qualify for inclusion in this genus (Kilian and Biberstein, 1984). The PBP profiles of these three species are no more different than those of other members of the genus and thus these species cannot be excluded on the basis of their PBP profiles. Although it is generally accepted that $H$. somnus and $H$. agni represent a common species (personal communication, T. Inzana, Washington State University) isolated from cattle or sheep, respectively, our data suggest that these are two distinct species. It has been proposed that $H$. parahaemolyticus and $H$. paraphrohaemolyticus are haemolytic variants of $H$. parainfluenzae (Albritton, 1982); however, their PBP profiles support separate species designations. Of the 19 species tested, only $H$. avium and $H$. paragallinarum had identical patterns which places in question their separation into two species. The latter species has been implicated in respiratory disease of chickens whereas $H$. avium, isolated from the respiratory tract of poultry, has not been associated with disease (Kilian and Biberstein, 1984).

Only the PBPs of $H$. influenzae have previously been characterised; their PBP expression has been shown to be growth-phase dependent and certain variability in binding, especially among ampicillinresistant non- $\beta$-lactamase producing isolates, has been noted (Mendelman and Chaffin, 1985; Mendelman et al., 1987). To minimise this possible variability (if it exists within the other species), we confined our analysis to penicillin susceptible Haemophilus isolates examined in the exponential phase of growth. The distinct profiles appear to reflect real PBP differences between species within the genus Haemophilus. However, as we have examined only single isolates, PBP variability within a species remains to be determined. Further analysis is needed to determine the exact number of PBPs for each species and the kinetics of penicillin acylation.

This work was supported by Public Health Service Grant AI 24630 from the National Institute of Health and by biomedical research support grant RR 005655 from the Children's Hospital and Medical Center, Seattle. We thank Dr Carla Clausen for review of the manuscript and Kae Pierce for excellent secretarial assistance. aegyptius bacteraemia in Brazilian Purpuric Fever. Lancet 2: 761-763.

Carlone G M, Sottnek F O, Plikaytis B D 1985 Comparison of outer membrane protein and biochemical profiles of Haemophilus aegyptius and Haemophilus influenzae biotype III. Journal of Clinical Microbiology 22: 708-713. 
Casin I, Grimont F, Grimont P A D 1986 Deoxyribonucleic acid relatedness between Haemophilus aegyptius and Haemophilus influenzae. Annals of Institute Pasteur/Microbiology 137b: 155-163.

Georgopapadakou N H, Liu F I 1980 Penicillin-binding proteins in bacteria. Antimicrobial Agents and Chemotherapy 18: 148157.

Kilian M, Biberstein E L 1984 Genus II. Haemophilus In: Holt J G (ed), Bergey's Manual of systematic bacteriology, vol. 1, The Williams and Wilkins Co., Baltimore, MD, p 558.

Martel A Y, Sottnek F O, Thomas M L, Albritton W L 1985 Susceptibility of Haemophilus aegyptius to trooleandomycin: lack of taxonomic value. Canadian Journal of Microbiology 32: 289-293.

Mendelman P M 1987 Haemophilus infections. In: Ballows A, Hausler W J (eds), Diagnostic procedures for bacterial, mycotic and parasitic infections, 7th edn. American Public Health Association, Inc., Washington DC, pp 301-316.

Mendelman P M, Chaffin D O 1985 Two penicillin binding proteins of Haemophilus influenzae are lost after cells enter stationary phase. FEMS Microbiology Letters 30: 399-402.
Mendelman P M, Chaffin D O, Musser J M, DeGroot R, Serfass D A, Selander R K 1987 Genetic and phenotypic diversity among ampicillin-resistant, non- $\beta$-lactamase-producing, nontypeable Haemophilus influenzae isolates. Infection and Immunity 55: 2585-2589.

National Committee for Clinical Laboratory Standards 1985 Methods for dilution antimicrobial susceptibility tests for bacteria that grow aerobically; approved standard. NCCLS publication m7-A. NCCLS, Villanova, PA.

O'Callaghan C H, Morris A, Kirby S M, Shingler A H 1972 Novel method for detection of $\beta$-lactamases by using a chromogenic cephalosporin substrate. Antimicrobial Agents and Chemotherapy 1 : 283-288.

Ohya S, Yamazaki M, Sugawara S, Matsuhashi M 1979 Penicillin-binding proteins in Proteus species. Journal of Bacteriology 137: 474-479.

Serfass D A, Mendelman P M, Chaffin D O, Needham C A 1986 Ampicillin resistance and penicillin-binding proteins of Haemophilus influenzae. Journal of General Microbiology 132: $2855-2861$. 